\section{Wenn selbstbestimmtes Sterben normal wird}

\section{Sterben alter Menschen als Herausforderung für Betroffene,} Nahestehende und Mitarbeitende in Altersinstitutionen ${ }^{1}$

Heinz Rüegger

Die Möglichkeiten der modernen Medizin, Menschen in kritischen Situationen noch am Leben zu erhalten und den Tod hinauszuschieben, sind beeindruckend. Es wird viel getan, um Leben zu retten; schliesslich ist das doch der Auftrag der Medizin. Und die meisten von uns nehmen diese Möglichkeiten lebensverlängernder Interventionen im Ernstfall auch gerne in Anspruch, selbst in weit fortgeschrittenem Alter, wenn Hochaltrigkeit durch mancherlei körperliche, psychische und soziale Belastungen ihren Tribut fordert. Der Wille zum Leben ist bei den meisten von uns stark ausgeprägt - offenbar eine Überlebensstrategie aus frühen Zeiten der evolutionären Menschheitsentwicklung. Entsprechend nimmt die durchschnittliche Lebenserwartung massiv zu. Die demografische Entwicklung der Bevölkerung ist eine beeindruckende zivilisatorische Erfolgsgeschichte. Alter und Sterben werden immer weiter hinausgeschoben ans Ende eines langen Lebens.

Die Errungenschaften moderner Medizin zur Erhaltung und Verlängerung des Lebens rufen allerdings ambivalente Gefühle hervor. Sie lösen mitunter starke Ängste aus. Ängste, dass einen die moderne Medizin daran hindere, zu gegebener Zeit in Ruhe sterben zu können. Ängste, das eigene Sterben werde technologisch verfremdet und fremdbestimmt durch eine

1 Die folgenden Ausführungen gehen auf einen Vortrag zurück, der an der 12. Fachtagung «Palliative Geriatrie» am 6. Oktober 2017 in Berlin gehalten wurde. Der Inhal verdankt sich dem gemeinsamen Gespräch mit Dr. med. Roland Kunz, Chefarzt der Universitären Klinik für Akutgeriatrie im Stadtspital Waid, Zürich.
High-Tech-Medizin, die einen auf einer Intensivstation künstlich überleben lasse, auch wenn die Lebensverlängerung vor allem zu einer Leidensverlängerung wird. Aus solchen Ängsten entwickelte sich der Ruf nach «selbstbestimmtem Sterben; das Recht jedes Menschen auf seinen eigenen Tod wurde postuliert und eingefordert.

Ist in den Medien oder generell in der Öffentlichkeit von selbstbestimmtem Sterben die Rede, geht es meist um die Frage nach dem assistierten Suizid. Damit wird die eigentliche Herausforderung des Themas aber verkannt. Assistierte Suizide machten selbst in der Schweiz mit ihrer in dieser Sache liberalen Gesetzgebung im Jahre 2014 nur gerade 1,2 \% aller Todesfälle aus, ${ }^{2}$ spielen darum für die Frage des selbstbestimmten Sterbens nur eine marginale Rolle.

\section{Der Tod kommt nicht mehr von selbst}

Die wesentlichen Fragen ergeben sich aus dem Umstand, dass wir in einer Zeit leben, in der wir aufgrund medizinischer Fortschritte und der Bedingungen unseres Gesundheitswesens genötigt sind, uns mit einem neuen Paradigma im Umgang mit dem Sterben vertraut zu machen. ${ }^{3}$ Dieses besteht darin, dass Sterben - früher Inbegriff der Erfahrung eines fremdverfügten Schicksals - zunehmend zum Gegenstand eigenen Entscheidens, also eines selbstbestimmten «Machsals〉 (Odo Marquard) wird. Angesichts des heute zur Verfügung stehenden medizinischen Arsenals an lebensverlängernden Massnahmen wird Sterben immer mehr zu einer Konsequenz

2 Bundesamt für Statistik, Assistierter Suizid (Sterbehilfe) und Suizid in der Schweiz. Todesursachenstatistik 2014 (BFS Aktuell 14: Gesundheit), Neuchâtel 2016, 1.

3 Roland Kunz / Heinz Rüegger, Selbstbestimmtes Ableben. Ein neues kulturelles Paradigma des Sterbens, in: Neue Zürcher Zeitung (NZZ) vom 12.04.17, 12.
Jahrbuch Diakonie Schweiz 3 (2019) - ISSN 2504-3994

Dieser Text ist lizenziert unter einer Creative Commons Namensnennung 4.0 International Lizenz (CC BY 4.0): (https://creativecommons.org/licenses/by/4.0/).
Jahrbuch Diakonie Schweiz 3 (2019) http://dx.doi.org/10.22018/JDS.2019.8 
von Therapieentscheidungen. Der Tod ist immer weniger Folge eines unerwarteten Schicksalsschlages, sondern immer häufiger Konsequenz einer bewussten Entscheidung, medizinischen Interventionen mit dem Ziel der Lebensverlängerung Grenzen zu setzen.

Nach neusten Untersuchungen erfolgt das Sterben in 58,7 \% der medizinisch begleiteten Todesfälle in der Schweiz erst, nachdem entsprechende Entscheidungen (sog. medical end-of-life decisions) gefällt wurden. ${ }^{4} \mathrm{Im}$ Fokus stehen dabei Massnahmen passiver Sterbehilfe, also des Verzichts auf lebensverlängernde Massnahmen. Der Lausanner Palliativmediziner Gian Domenico Borasio nimmt sogar an, dass dies in der Schweiz für 75 $\%$ der Sterbefälle zutrifft. ${ }^{5}$

Nun stellt sich natürlich die Frage, wem es zusteht, entsprechende Entscheidungen zu fällen. Ethisch gesehen kann es da nur eine Antwort geben: Der Entscheid muss bei der betroffenen Person selbst liegen, weil nach dem Prinzip der Patientenautonomie nur ihr das Recht zukommt, über medizinische Eingriffe am eigenen Leib zu befinden und sie als zulässig oder unzulässig zu erklären. Und wenn sie nicht mehr selbst entscheiden kann, müssen vertretungsberechtigte Personen bestimmen, was der mutmassliche Wille der Patientin ist. Dieser ist dann für das medizinische Personal therapieentscheidend und verbindlich. ${ }^{6}$

4 Georg Bosshard / Samia A. Hurst / Milo Alan Puhan, Medizinische Entscheidungen am Lebensende sind häufig, Swiss Medical Forum - Schweizerisches Medizin-Forum 16 (42), 2016, 896-898; Georg Bosshard u. a., Medical End-of-Life Practices in Switzerland. A Comparison of 2001 and 2013, JAMA Internal Medicine 176 (4), 2016 555f; Margareta Schmid u. a., Medical end-of-life decisions in Switzerland 2001 and 2013: Who is involved and how does the decision-making capacity of the patient impact?, Swiss Medical Weekly 2016; 146 (doi:10.4414/smw.2016.14307).

5 Gian Domenico Borasio, Selbst bestimmt sterben. Was es bedeutet. Was uns daran hindert. Wie wir es erreichen können, München 2014, 41.

6 Stefanie Haussener, Entscheidungen am Lebensende im Spannungsfeld zwischen Selbst- und Fremdbestimmung, Patientenwillen und -wohl. Eine kritische Analyse von
Das heisst: In der Mehrzahl der Fälle ist Sterben heute nicht mehr einfach eine Entscheidung der Natur, des Schicksals, des Arztes oder des 〈Herrn über Leben und Tod». Wir müssen den Tod heute immer häufiger in die eigenen Hände nehmen, auch wenn wir keinen Suizid begehen wollen. Der Tod kommt nicht mehr einfach ungefragt auf uns zu wie der Sensemann in unzähligen Bildern der Kunstgeschichte - er muss heute vom Menschen selbst beschlossen, geplant und durchgeführt werden. Wir müssen heute immer mehr selbst entscheiden, was früher dem Schicksal überlassen wurde. Wir müssen uns bewusst entscheiden, wann wir eine Therapie nicht mehr in Anspruch nehmen oder sie abbrechen wollen. Das Sterben lassen wir nicht mehr einfach geschehen. Für das Sterben - oder alternativ dazu für das Weiterleben - muss man sich entscheiden. Die Frage ist dabei gar nicht, ob wir das gut finden oder nicht. Es ist einfach so, is Teil der Rahmenbedingungen des Sterbens in der heutigen Medizin und in unserem heutigen Gesundheitswesen.

Der Soziologe Reimer Gronemeyer hält pointiert fest: «Das ist das Neue: Sterben und Tod sind für uns moderne Menschen zum «Problem geworden. Der Tod kommt nicht mehr, sondern er wird zur letzten Gestaltungsaufgabe des Menschen.» Und weiter: «Sterben wird planbar - das ist ein Grundzug der modernen Gesellschaft.» ${ }^{7}$

Das heisst: Wir haben es heute im Blick auf die Selbstbestimmung beim Sterben mit einem kulturgeschichtlich neuen Paradigma des Sterbens zu tun - weit über das zahlenmässig begrenzte Phänomen assistierter Suizide hinaus. Das Sterben eines Menschen wird zunehmend an sein Sich-Entscheiden-zum-Sterben gekoppelt. Das ist eine neue Situation. In früheren

Rechtslage und medizinischer Praxis, Jusletter 30. Januar 2017; Regina Aebi-Müller, Der urteilsunfähige Patient - eine zivilrechtliche Auslegeordnung, Jusletter 22. September 2014.

7 Reimer Gronemeyer, Sterben in Deutschland. Wie wir dem Tod wieder einen Platz in unserem Leben einräumen können, Frankfurt a. M. 2007, 37. 
Jahrhunderten war die Verbindung zwischen Sterben und Sich-Entscheiden-zum-Sterben Charakteristikum des Suizids (oder des 〈Selbstmords, wie man es früher nannte). Und dieser galt als Sündenfall par excellence, den die Gesellschaft wie kaum etwas anderes mit allen möglichen religiösen, sozialen und strafrechtlichen Verboten zu verhindern bestrebt war. Ausgerechnet diese während Jahrhunderten so heftig verworfene Verknüpfung von Sterben und Sich-Entscheiden-zum-Sterben wird heute auf dem Hintergrund neuer medizinischer Errungenschaften zu einem notwendigen Grundzug des Sterbens in unserem modernen, medizinisch hoch gerüsteten Gesundheitswesen.

Die Freiheit zur Selbstbestimmung im Blick auf das eigene Sterben ist heute ein zentraler ethischer wie juristischer Grundsatz, insbesondere in der Geriatrie. ${ }^{8}$ Mit diesem neuen Paradigma des Sterbens angemessen umzugehen, ist für Betroffene, für die den Sterbeprozess begleitenden Angehörigen sowie für professionelle Mitarbeitende (Ärztinnen, Pflegende, Seelsorgende) allerdings noch weithin ungewohnt und anspruchsvoll.

8 Martin Teising ist zuzustimmen: «Die Selbstbestimmung des Einzelnen in persönlichen existenziellen Dingen hat sich zum höchsten ethischen Wert entwickelt.» Im Gefolge davon gilt: «Der aufgeklärte Zeitgenosse soll auch sein eigenes Lebensendszenarium selbst kontrollieren. Er sieht sich zur Selbstinszenierung einschliesslich der Gestaltung des eigenen Todes gedrängt.» Teising spricht dabei geradezu von einer «Fetischisierung individueller Autonomie» (Martin Teising, Selbstbestimmung zwischen Wunsch und Illusion. Eine psychoanalytische Sicht, Göttingen 2017, 11-56.13). - Zu Rechtslage in der Schweiz vgl. den Bundesgerichtsentscheid 133 I 58 vom 03.11.06, der festhält: «Zum Selbstbestimmungsrecht im Sinne von Art. 8 Ziff. 1 EMRK gehört auch das Recht, über Art und Zeitpunkt der Beendigung des eigenen Lebens zu entscheiden».

\section{Freiheit oder Überforderung?}

Jedem Individuum wird heute ein Mass an Selbstbestimmung im Blick auf sein Sterben zuerkannt, das früher nicht üblich war. Die Forderung nach «selbstbestimmtem Sterben, nach dem «Recht auf den eigenen Tod» setzt sich heute immer mehr durch. Selbstbestimmtes Sterben wandelt sich dabei von einem eingeforderten Anspruch (Stichwort: Recht auf den eigenen Tod) zu einer zugemuteten Verpflicbtung (Stichwort: selber über lebensverlängernde Massnahmen entscheiden müssen). Das Individuum steht heute gewollt oder ungewollt vor der Herausforderung, sich mit dieser Freiheit auseinander zu setzen und sie zu leben.

Mit dieser Freiheit ist allerdings auch die Selbstverantwortung des Individuums im Blick auf das eigene Sterben gewachsen. Wenn Sterben von unserem Entscheiden abhängt, sind wir für diese Entscheidung moralisch verantwortlich. Es kommt zu so etwas wie einer Responsibilisierung oder Moralisierung des Sterbens bzw. des Noch-nicht-sterben-Wollens. Mit den Worten von Reimer Gronemeyer: «Solange der Tod «kam» musste sich keiner rechtfertigen: Es bedurfte einer solchen Debatte nicht. Das moderne Subjekt, der homo modernissimus, hat sich in die fatale Lage gebracht, dass es nun selbst sein Sterben und seinen Tod zu verantworten hat.»'

Solche Entscheidungen können schwierig sein, ja an eine Überforderung grenzen. Manch ein Sterbender dürfte höchst ambivalent vor heute anstehenden medical end-of-life decisions stehen und sich schwer damit tun, selbstbestimmt zu entscheiden. Es ist eben durchaus nicht so, dass wirwie sich das eine abstrakte Ethik theoretisch vielleicht zurecht legen mag - grundsätzlich fähig sind, autonom zu entscheiden, was wir wollen. Gerade in extremen Situationen, in denen existenzielle Fragen um Leben und Tod auf dem Spiel stehen, sind Menschen oft hin und her gerissen zwischen unterschiedlichen Optionen und brauchen Zeit, um Klarheit dar-

9 Gronemeyer, Sterben in Deutschland (Anm. 7), 177.
Jahrbuch Diakonie Schweiz 3 (2019) - ISSN 2504-3994

CC by 4.0
Jahrbuch Diakonie Schweiz 3 (2019) http://dx.doi.org/10.22018/JDS.2019.8 
über zu gewinnen, was sie denn wirklich wollen, was für sie in der konkreten Situation und auf dem Hintergrund ihres bisherigen Lebensentwurfs stimmig ist.

Im Blick auf einen angemessenen Umgang mit Selbstbestimmung beim Sterben stellen sich konkrete Herausforderungen für uns alle als potenziell Betroffene, für involvierte Angehörige und für medizinisches Personal im Besonderen.

\section{Herausforderung für uns alle als potenziell betroffene Indi- viduen}

Als Bürger eines Landes mit einem modernen Gesundheitswesen und einer hochstehenden medizinischen Versorgung kommen wir nicht darum herum zu lernen, uns mit dem Gedanken ans eigene, selbstbestimmte Sterben auseinander zu setzen. Es geht darum, mindestens ein Stück weit zu klären, was uns im Blick auf unser irgendwann einmal bevorstehendes Sterben persönlich wichtig ist, was wir unbedingt möchten und was gerade nicht. Das gehört heute gleichsam zu den Aufgaben eines mündigen Bürgers - auch wenn das den meisten Zeitgenossen noch nicht bewusst sein dürfte.

Dazu ist es nötig, sich mit der eigenen Endlichkeit, dem eigenen «Sein zum Tode> (Martin Heidegger) existenziell zu beschäftigen, und zwar möglichst bevor wir uns in der terminalen Phase befinden. In der philosophischen und religiösen Tradition sprach man in diesem Zusammenhang während Jahrhunderten von einer ars moriendi, also von einer Lebenskunst des Sterbens oder vielleicht etwas besser ausgedrückt: einer Lebenskunst, sich mit der Endlichkeit des eigenen Lebens anzufreunden. ${ }^{10}$ Eine solche Lebenskunst kann auf verschiedene Weise eingeübt werden: im Medium

10 Heinz Rüegger, Das eigene Sterben. Auf der Suche nach einer neuen Lebenskunst, Göttingen 2006 . der Spiritualität, der Kunst oder durch konkrete Begegnungen mit hochbetagten und sterbenden Menschen. Auch ein Gespräch mit Fachleuten aus Medizin und Pflege kann nützlich sein, um sich realistische Vorstellungen über Möglichkeiten des Sterbens im heutigen Gesundheitswesen zu machen. Nicht zuletzt kann das Ausfüllen einer Patientenverfügung im Gespräch mit Angehörigen Anregungen bieten, sich mit diesen Fragen zu beschäftigen - Fragen, die zugegebenermassen für viele nicht leicht anzusprechen sind.

Das aktive Mitbestimmen und Mitgestalten des eigenen Sterbens ist jedoch nur die eine Seite der Medaille, die es heute aufgrund der Entwicklungen in der modernen Medizin und in unserem Gesundheitswesen allerdings verstärkt ins Bewusstsein zu bringen gilt. Zu einem reifen, humanen Umgang mit dem Sterben gehört auch die existenzielle Fähigkeit, Dinge mit sich geschehen zu lassen, sich aus der Hand zu geben und anzunehmen, dass es Entwicklungen gibt, die sich unserer Kontrolle entziehen Das ist die andere Seite der Medaille, die herkömmlicherweise stark betont wurde und die heute angesichts des starken Akzents auf der Selbstbestimmung beim Sterben in ihrer Bedeutung etwas unterschätzt werden dürfte. Im Ernstnehmen dieser passiven Seite menschlichen Lebens können sich durchaus auch Dimensionen von Freiheit eröffnen: Freiheit, nicht zu meinen, man müsse auch im Sterben noch alles unter Kontrolle haben, damit das eigene Sterben als ein 〈Sterben in Würde` anerkannt werde.

Eine solche Lebenseinstellung des Hinnehmens kann gefördert werden durch das Einüben einer Haltung der Gelassenheit oder einer Spiritualität des Gott-Vertrauens, durch das Entwickeln eines Gefühls von Kohärenz und Sinnhaftigkeit im Sinne der Salutogenese von Aaron Antonovsky $^{11}$ oder durch die Stärkung der Resilienz, also der psychischen Widerstandskraft im Umgang mit Herausforderungen des Schicksals.

11 Aaron Antonovsky, Salutogenese. Zur Entmystifizierung der Gesundheit (Forum für Verhaltenstherapie und psychosoziale Praxis, Bd. 36), Tübingen 1997. 
Beide Aspekte - das eigenverantwortliche Gestalten (Selbstbestimmung) und das passive Hinnehmen (Geschehenlassen) - gehören zu einem humanen, selbstbestimmten Sterben jenseits von Verantwortungslosigkeit und Kontrollzwang. Dem Philosophen Wilhelm Schmid ist zuzustimmen: «Selbstbestimmung ist ein aktiver ebenso wie ein passiver Prozess, ein Tun ebenso wie ein Hinnehmen und Lassen, ein eigenes Gestalten wie auch ein Sich-gestalten-Lassen von anderen, von Umständen und Situationen.» ${ }^{12}$ Hier eine gute Balance zu finden zwischen beiden Aspekten, scheint mir heute eine zentrale Herausforderung für das Individuum im Blick auf sein bevorstehendes Sterben zu sein.

\section{Herausforderungen für Angehörige}

Jeder und jede stirbt seinen bzw. ihren eigenen Tod. Aber unser Sterben geht nicht nur uns selber an, sondern betrifft auch unsere Angehörigen, also diejenigen Menschen, mit denen wir besonders verbunden sind, vor allem diejenigen, die uns einmal im Prozess des Sterbens persönlich begleiten. Ihr Begleiten kann hilfreich oder schwierig sein.

Hilfreich ist es dann, wenn Angehörige einerseits bereit sind, sich offen auf das Phänomen des Sterbens einzulassen, mit allem, was das in ihnen selbst auslöst. Wenn sie also das Thema Sterben und Tod nicht verdrängen oder überspielen. Hilfreich sind Angehörige andrerseits, wenn sie ihre eigenen Wünsche, Meinungen und Wertungen zurücknehmen und sich ganz darauf fokussieren, dem Sterbenden als Gesprächspartner zu helfen, durch eine mögliche Vielzahl von ambivalenten Empfindungen hindurch eine Haltung und Entscheidung herauszubilden, die seinem autonomen Willen entsprechen und für ihn - nicht notwendigerweise auch für die Angehörigen! - stimmig ist.

12 Wilhelm Schmid, Mit sich selbst befreundet sein. Von der Lebenskunst im Umgang mit sich selbst, Frankfurt a.M. 2004, 119
Eine solche Rolle einzunehmen, dem Sterbenden also nicht gutmeinend die eigene Meinung aufzudrängen - als wüsste man, was für ihn in der gegebenen Situation gut und richtig ist -, sondern ihm in einem geduldigen, nicht-direktiven Dialog zu helfen, sich über sich selbst Klarheit zu verschaffen und der Herausforderung eines selbstbestimmten Sterbens überhaupt gewachsen zu sein, das ist nicht einfach, sondern fordert von Angehörigen ein beachtliches Mass an selbstkritischer Differenziertheit.

Nur allzu häufig ist festzustellen, dass Angehörige Sterbende zum Beispiel dahingehend zu beeinflussen versuchen, dass sie noch nicht sterben sollen - weil die Angehörigen damit ein Problem haben und sich diesem Problem nicht ehrlich stellen wollen oder können. Es gehört mit zu den Aufgaben professioneller Sterbebegleiter (Ärzte, Pflegende, Seelsorgende), Angehörigen zu helfen, mit solchen Ängsten umzugehen, sich auf die Realität des Sterbens einzulassen und dem Sterbenden so offen zu begegnen, dass er herausfinden kann, was für ibn ein - soweit möglich selbstbestimmtes Sterben ist.

\section{Herausforderungen für professionell Begleitende}

Für medizinisches Personal stellt sich die Herausforderung, sterbende Menschen so zu begleiten, dass das Lebensende nicht immer weiter medikalisiert wird. Es geht um die Reduzierung des «medizinischen Lärms», um Raum und Ruhe zu schaffen, damit die eigene Lebenssinfonie zu Ende geschrieben werden kann. Dabei kommt einer ganzheitlichen Betreuung und insbesondere einem kompetenten Schmerz- und Symptommanagement entscheidende Bedeutung zu. All dies muss im Zeichen einer radikalen Patientenorientierung erfolgen, die den betroffenen Menschen mit seinem ganz persönlichen Erleben und seinen subjektiven Wünschen und Prioritäten in den Mittelpunkt stellt. Gerade dadurch wird Palliative Care eine Hilfe zu selbstbestimmtem Leben und Sterben.

Eine Aufgabe, die der Ärzteschaft in Zukunft vermehrt zufallen wird, besteht darin, Patienten rechtzeitig darüber zu informieren, wie man im Rahmen des heutigen Gesundheitswesens überhaupt sterben kann. Der
Jahrbuch Diakonie Schweiz 3 (2019) - ISSN 2504-3994

CC by 4.0
Jahrbuch Diakonie Schweiz 3 (2019) http://dx.doi.org/10.22018/JDS.2019.8 
Hastings-Report von 1996, das letzte grosse internationale Projekt einer Verständigung über die Ziele der Medizin, weist dieser unter anderem die Aufgabe zu, Menschen zu einem möglichst friedlichen Sterben zu verhelfen (to pursue a peaceful death). ${ }^{13}$ Dazu gehört eine angemessene Aufklärung darüber, welche Optionen bestehen, sein Leben möglichst ruhig und zu einem subjektiv wünschbaren Zeitpunkt zu beenden (im Sinne einer Patientenedukation). Eine 2016 veröffentlichte Studie des Berner LINK-Instituts zeigt, dass $72 \%$ der Befragten frühzeitig (also nicht erst bei nahendem Lebensende!) von ihrem Arzt über Möglichkeiten der Selbstbestimmung am Lebensende orientiert werden möchten; ferner erwarten $66 \%$ dass sie ihre Ärztin bei nahendem Lebensende über verschiedene Möglichkeiten des Sterbens aufklärt. ${ }^{14}$ Nur wer um solche Optionen und Alternativen weiss, kann mündig und selbstbestimmt entscheiden. An einer offenen, rücksichtsvollen Gesprächskultur über das Sterben zu arbeiten, bleibt darum eine zentrale Forderung an die Aus- und Fortbildung des ärztlichen Personals.

Der amerikanische Medizinethiker Daniel Callahan hat darauf hingewiesen, dass viele Menschen und ihre Ärzte die Tendenz haben, potenziell tödlich verlaufende Krankheiten immer wieder durch kurative Interventionen zu bekämpfen in der Annahme, ein späteres Sterben in fortgeschrittenerem Alter sei besser, friedlicher, sinnvoller und die einem potenziell früheren Sterben abgetrotzte zusätzliche Lebenszeit sei es Wert, den Tod

13 Zum Hastings-Report auf Deutsch siehe: Die Ziele der Medizin - neue Prioritäten setzen, in: Werner Stauffacher / Johannes Bircher (Hg.), Zukunft Medizin Schweiz. Das Projekt «Neu-Orientierung der Medizin» geht weiter, Basel 2002, 324-389. Ziel 4 lautet: «Verhinderung eines vorzeitigen Todes und das Streben nach einem friedvollen Tod» (ebd. 327).

14 Link-Institut, Letzter Lebensabschnitt. Was erwartet die Bevölkerung vom Arzt?, URL: https://www.exit.ch/fileadmin/user_upload/files/Studie_LINK_Institut_Erwartungen_an_Aerzte_2016.pdf (abgerufen am 17.01.19). immer noch mehr hinauszuschieben. ${ }^{15}$ Und dann beklagen sich Betroffene, dass ihr Leben in hohem Alter mit der damit einhergehenden Multimorbidität so beschwerlich sei und das nun erst viel später erfolgende Sterben unter Umständen mühsam und langwierig sei!

«Der Herrgott hat das irgendwie nicht gut eingerichtet», sagte mir eine hochbetagte Frau in einer solchen Situation. Sie übersah dabei allerdings, dass das mit dem Herrgott gar nichts zu tun hat, sehr wohl aber mit ihrem eigenen bisherigen Umgang mit Krankheiten, die sie konsequent durch kurative, lebensverlängernde Massnahmen, also selbst bestimmt, bekämpfte. Auf diese Weise beraubte sie sich zahlreicher Möglichkeiten, durch die sie relativ friedlich ihr Leben hätte beenden können, bevor es für sie zu einer immer unerträglicher werdenden Belastung wurde. Aber auf einen solchen Gedanken war sie selbst nie gekommen. Über einen solchen Gedanken hat auch nie jemand mit ihr gesprochen: ihre Angehörigen nicht, ihre Ärzte nicht und ihr Seelsorger nicht. Auch professionell Begleitende sind in der Regel nicht darauf vorbereitet, solche Gespräche auf eine zugleich fachlich kompetente und menschlich einfühlsame Art zu führen. Aber eben solche Aufklärung über relativ friedliche Arten des Sterbens und Ermutigung zu einem rechtzeitigen Zulassen des Sterbens sieht Callahan als genuine Aufgabe der Ärzteschaft gegenüber ihren Patienten.

15 Daniel Callahan gibt zu bedenken: «With each serious illness, the question should be raised whether this illness should be allowed to proceed and become the cause of death. The present way of managing the critically ill is ordinarily just the opposite, treating each potentially fatal illness as if it were reversible, and as if it must be reversed. Instead of thinking, as I propose, that this death now may be better than another death later, the modern way is always to prefer the later, different, death over the present possibiliy. (...) There is no reason whatever to believe that a later death from an as yet unknown cause will be better than a death from a present known cause... I am suggesting instead that, once a potentially fatal illness appears, it be considered seriously as the candidate for the cause of death, if other conditions of the timing and circumstances of death are acceptable» (Daniel Callahan, The Troubled Dream of Life. In Search of a Peaceful Death, Washington 2000, 198f.). 
Fazit

Selbstbestimmtes Sterben wird immer mehr zum neuen Paradigma unseres Umgangs mit Sterben und Tod unter den real existierenden Bedingungen unseres heutigen Gesundheitswesens. Darauf sind wir mentalitätsmässig und kulturgeschichtlich noch kaum vorbereitet. Soll dieses neue Paradigma als Zugewinn an Freiheit und Selbstbestimmung positiv gelebt werden können, sind wir alle - unmittelbar vom Sterben Betroffene, Angehörige und professionell Begleitende - herausgefordert, uns ehrlich mit dem Sterben auseinanderzusetzen, es als elementaren Teil unseres Lebens anzuerkennen und darüber miteinander in ein offenes Gespräch zu treten. Davon sind wir derzeit in vielen Situationen noch ziemlich weit entfernt. Es besteht Handlungsbedarf, oder vielmehr: Lernbedarf.

Letztlich geht es darum, in Aufnahme einer langen abendländischen Tradition als Teil einer ars vivendi, einer Lebenskunst, wieder so etwas wie eine ars moriendi, eine Kunst des Sterbens, oder besser: des sich Anfreundens mit der eigenen Endlichkeit zu entwickeln. ${ }^{16}$ Norbert Bolz hat Recht, wenn er feststellt: «Endlichkeit muss man lernen.» ${ }^{17}$ Auch Sterben-Können muss man lernen in einer Kultur, die den Tod medizinisch immer weiter hinausschieben kann und ihn so ein gutes Stück weit zum Gegen stand eigenen Entscheidens gemacht hat.

Autor:

Heinz Rüegger, Dr. theol., freischaffender Theologe, Ethiker und Gerontologe sowie freier Mitarbeiter im Institut Neumünster, einem auf Fragen des Alters spezialisierten interdisziplinären Kompetenzzentrum der Stiftung Diakoniewerk Neumünster, Zollikerberg

16 Rüegger, Das eigene Sterben (Anm. 10).

17 Norbert Bolz, Das Wissen der Religion. Betrachtungen eines religiös Unmusikalischen, Paderborn 2008, 128 